\title{
Nonnegative Solutions of a Nonlinear System and Applications to Elliptic BVPs*
}

\author{
Guangchong Yang ${ }^{1}$ and Yanqiu Chen ${ }^{2}$
}

\begin{abstract}
In this communication, we study the existence of nonnegative solutions of a nonlinear system in Banach spaces. These maps involved in the system defined on cone do not necessarily take values in the cone. Using fixed point theorems just established for this type of mappings, nonnegative solutions of the system are obtained and used to investigate elliptic boundary value problems (BVPs).
\end{abstract}

MSC(2010): 47H10, 35J57.

Keywords: Nonlinear system, Nonnegative solutions, Nowhere normal-outward maps, Fixed point, Elliptic BVPs.

\footnotetext{
${ }^{1}$ College of Applied Mathematics, Chengdu University of Information Technology, Chengdu, Sichuan 610225, P. R. China.

${ }^{2}$ College of Applied Mathematics, Chengdu University of Information Technology, Chengdu, Sichuan 610225, P. R. China.
} 


\section{Introduction}

It has been well-known that nonlinear systems arise in many fields of scientific researches and engineering practices, the study on existence of nonnegative solutions for that is very interesting and of great importance, see, for example, [1, $2]$ and the references there. However, the nonlinear terms involved in that take negative values in many cases, to the best of our knowledge, there is little study on it.

Recently, Yang, one of authors of [3], and Lan established a new fixed point index theory for nowhere normal-outward compact maps [4] and proved a few fixed point theorems, which were used to population models with sign-changing nonlinearities [5]. In this communication, we expand some results [3] to systems and apply them to investigate elliptic boundary value problems (BVPs).

We recall some knowledge on $r$-nowhere normal-outward maps and a fixed point theorem for these maps obtained in [3].

Let $K$ be a closed convex set in a Banach space $X$ with norm $\|\cdot\|$, and let $r: X \rightarrow K$ be a retraction, that is, $r$ is continuous and satisfies $r(y)=y$ for $y \in K$. Recall that a map $A: D \subset K \rightarrow X$ is called to be a $r$-nowhere normal -outward map on $D$ relative to $K$ if

$$
A x \in\left(X \backslash r^{-1}(x)\right) \cup\{x\} \text { for } x \in D .
$$

The following criterion was obtained in [3, Proposition 2.1].

Lemma 1.1 Let $A: D \subset K \rightarrow X$ be a map and let $r: X \rightarrow K$ be a retraction. If

$$
x=A(r(x)) \text { for some } x \in r^{-1}(D)
$$

implies $x \in D$, then $A$ is a $r$-nowhere normal-outward map on $D$ relative to $K$. A map $A: D \subset X \rightarrow X$ is said to be compact if $A$ is continuous and $A(S)$ is relatively compact for each bounded subset $S$ of $D$.

Let $D_{K}=D \cap K, \bar{D}_{K}=\bar{D} \cap K$ and $\partial D_{K}=\partial D \cap K$. In [3], we established the following known result (see [3, Theorem 3.2]).

Theorem 1.1 Let $K$ be a closed convex set in $X$ and let $r: X \rightarrow K$ be a retraction. Let $D^{1}, D$ be bounded open sets in $X$ such that $\overline{D_{K}^{1}} \subset D_{K}$ and $D_{K}^{1} \neq \varnothing$. Assume that $A: \bar{D}_{K} \backslash D_{K}^{1} \rightarrow X$ is compact such that the following conditions hold.

$\left(h_{1}\right)$ There exists $x_{0} \in D_{K}$ such that $t A+(1-t) \widehat{x_{0}}$ is r-nowhere normaloutward on $\bar{D}_{K}$ relative to $K$ for $t \in(0,1]$.

$$
\text { (LS) } x \neq t A x+(1-t) x_{0} \text { for } x \in \partial D_{K} \text { and } t \in(0,1) \text {. }
$$


$\left(h_{2}\right)$ There exists $e \in K \backslash\{0\}$ such that $A+\lambda e$ is a r-nowhere normaloutward on $\overline{D_{K}^{1}}$ relative to $K$ for $\lambda>0$.

(E) $x \neq A x+\lambda e$ for $x \in \partial D_{K}^{1}$ and $\lambda \geq 0$.

Then $A$ has a fixed point in $\bar{D}_{K} \backslash \bar{D}_{K}^{1}$.

Let $K$ be a cone in $X$. Then $K$ defines a partial order $\leq$ in $X$ by $x \leq y$ if and only if $y-x \geq 0$. A cone $K$ is said to be reproducing if $X=K-K$, to be total if $X=\overline{K-K}$ and to be normal if there exists $\sigma>0$ such that $0 \leq x \leq y$ implies $\|x\| \leq \sigma\|y\| \quad[1]$.

Recall that a real number $\lambda$ is called an eigenvalue of a linear operator $L: X \rightarrow X$ if there exists $\varphi \in X \backslash\{0\}$ such that $\lambda \varphi=L \varphi$. The radius of the spectrum of $L$ in $X$, denoted by $r(L)$, is given by $r(L)=\lim _{m \rightarrow \infty} \sqrt[m]{\|L\|^{m}}$.

We write

$$
\mu_{1}(L)=\frac{1}{r(L)} .
$$

We denote by $L(K)$ the set of compact linear operators $L: X \rightarrow X$ satisfying $L(K) \subset K$ and $r(L)>0$. By Krein-Rutman theorem (see [6, Theorem 3.1] or [7]), if $K$ is a total cone and $L \in L(K)$, then there exists an eigenvector $\varphi \in K \backslash\{0\}$ such that

$$
\varphi=\mu_{1}(L) L \varphi
$$

\section{Nonnegative solutions of a nonlinear system}

Let $n \geq 2, K^{n}=K \times K \times \ldots \times K$ and $A_{i}: K^{n} \rightarrow X$. We shall establish the existence results of nonzero solutions in $K^{n}$ for the following system of the form

$$
x_{i}=A_{i}(x) \text { for } i \in I_{n}:=\{1,2, \ldots, n\},
$$

where $x=\left(x_{1}, x_{2}, \ldots, x_{n}\right) \in X_{n}$.

Let $X_{n}=X \times X \times \ldots \times X$ with the maximum norm

$$
\|x\|=\max \left\{\left\|x_{1}\right\|,\left\|x_{2}\right\|, \ldots,\left\|x_{n}\right\|\right\}
$$

and $r_{n} x=\left(r x_{1}, r x_{2}, \ldots, r x_{n}\right)$. Then $r_{n}: X_{n} \rightarrow K^{n}$ is a retraction. A map $A: K^{n} \rightarrow X$ is called to be a $r$-nowhere normal-outward map with respect to component $x_{i}\left(i \in I_{n}\right)$ on $K^{n}$ relative to $K$, if $x \in X_{n}, x_{i}=A_{i} r_{n} x$ implies $x_{i} \in K$. 
Using Theorem 1.1, we prove

Theorem 2.1 Let $K$ be a total and normal cone in $X$, and let $r: X \rightarrow K$ be a retraction. Assume that $A_{i}: K^{n} \rightarrow X$ is compact and satisfies the following conditions:

$\left(h_{n}\right)$ For any $i \in I_{n}$, there exists $x_{i} \in K$ such that $t A_{i}+(1-t) \widehat{x}_{l}$ is $r$-nowhere normal-outward map with respect to component $x_{i}$ on $K^{n}$ relative to $K$ for $t \in(0,1]$.

$(L S)_{n} \quad$ For any $i \in I_{n}$, there exist $v_{i} \in K \backslash\{0\}, \quad L_{i} \in \mathrm{L}(K)$ and $\varepsilon_{i} \in\left(0, \mu_{1}\left(L_{i}\right)\right)$ such that

$$
A_{i} x \leq\left(\mu_{1}\left(L_{i}\right)-\varepsilon_{i}\right) L_{i}\left(x_{i}\right)+v_{i} \text { for } x \in K .
$$

$(E)_{n}$ There exist $i_{0} \in I_{n}, \rho_{0}>0$ and $L_{0} \in \mathrm{L}(K)$ such that

$$
A_{i_{0}} x \geq \mu_{1}\left(L_{0}\right) L_{0}\left(x_{i_{0}}\right) \text { for } x \in \partial K_{\rho_{0}} .
$$

Then (2.1) has a solution in $K^{n} \backslash\{0\}$.

Proof. Let $A: K^{n} \rightarrow X_{n}$ be defined by $A x=\left(A_{1} x, A_{2} x, \ldots, A_{n} x\right)$. Then $A$ is compact since $A_{i}$ is compact. Let $x \in X_{n}$ such that $x=t \operatorname{Ar} x+(1-t) x_{0}$, where $x_{0}=\left(x_{1}, x_{2}, \ldots, x_{n}\right)$. Then $x_{i}=t A_{i} r x+(1-t) x_{i}$. By $\left(h_{n}\right)$, we see $x_{i} \in K$. Hence $t A+(1-t) \widehat{x_{0}}$ is a $r_{n}$-nowhere normal-outward map on $K^{n}$ relative to $K^{n}$ and $\left(h_{1}^{\prime}\right)$ of Theorem 1.1 holds.

Let $\rho>0, K_{\rho}^{n}=\left\{x: x \in K^{n},\|x\|<\rho\right\}$ and $\partial K_{\rho}^{n}=\left\{x: x \in K^{n},\|x\|=\rho\right\}$.

Since

$$
r\left(\left(\mu_{i}\left(L_{i}\right)-\varepsilon_{i}\right) L_{i}\right)=\left(\mu_{1}\left(L_{i}\right)-\varepsilon_{i}\right) r\left(L_{i}\right)<1,
$$

$\left(I-\left(\mu_{1}\left(L_{i}\right)-\varepsilon_{i}\right) L_{i}\right)^{-1}$ exists and is a bounded linear operator such that

$$
\left(I-\left(\mu_{1}\left(L_{i}\right)-\varepsilon_{i}\right) L_{i}\right)^{-1}(K) \subset K,
$$

where $I: X \rightarrow X$ is the identical mapping $I(z)=z$.

Let $\sigma$ be the normality constant of $K$ and

$$
\rho^{*}=\max \left\{\rho_{0}, \sigma\left\|\left(I-\left(\mu_{i}\left(L_{i}\right)-\varepsilon_{i}\right) L_{i}\right)^{-1}\left(v_{i}+x_{i}\right)\right\|, i \in I_{n}\right\} .
$$

Let $\rho>\rho^{*}$ and $x_{0}=\left(x_{1}, x_{2}, \ldots, x_{n}\right)$. Then $x_{0} \in K^{n}$. We prove that

$$
x \neq t A x+(1-t) x_{0} \quad \text { for } x \in \partial K_{\rho}^{n} \text { and } t \in(0,1] .
$$


In fact, if not, there exist $x \in \partial K_{\rho}^{n}$ and $t \in(0,1]$ such that $x=t A x+(1-t) x_{0}$. This, together with $(L S)_{n}$, implies

$$
\begin{aligned}
x_{i} & =l A_{i} x+(1-l) x_{i} \leq l\left[\left(\mu_{1}\left(L_{i}\right)-\varepsilon_{i}\right) L_{i}(x)+v_{i}\right]+(1-l) x_{i} \\
& \leq\left(\mu_{1}\left(L_{i}\right)-\varepsilon_{i}\right) L_{i}(x)+v_{i}+x_{i}
\end{aligned}
$$

and

$$
\left(I-\left(\mu_{i}\left(L_{i}\right)-\varepsilon_{i}\right) L_{i}\right) x_{i} \leq v_{i}+x_{i} \text { for } i \in I_{n} .
$$

This, together with $\left(I-\left(\mu_{i}\left(L_{i}\right)-\varepsilon_{i}\right) L_{i}\right)^{-1}(K) \subset K$, implies

$$
x_{i} \leq\left(I-\left(\mu_{i}\left(L_{i}\right)-\varepsilon_{i}\right) L_{i}\right)^{-1}\left(v_{i}+x_{i}\right) .
$$

Since $K$ is a normal cone with normality constant $\sigma$, it follows that

$$
\left\|x_{i}\right\| \leq \sigma\left\|\left(I-\left(\mu_{i}\left(L_{i}\right)-\varepsilon_{i}\right) L_{i}\right)^{-1}\left(v_{i}+x_{i}\right)\right\| \leq \rho^{*} \text { for } i \in I_{n} .
$$

Hence, we have $\rho=\|x\| \leq \rho^{*}<\rho$, a contradiction. $(L S)$ of Theorem 1.1 holds.

Without loss of generality, we may assume $A$ has not fixed point in $\partial P_{\rho_{0}}$ (otherwise, the result has been proved). Since $K$ is total, it follows from the KreinRutman theorem that there exists $\varphi_{0} \in K \backslash\{0\}$ such that

$$
\begin{aligned}
& \varphi_{0}=\mu_{1}\left(L_{0}\right) L_{0}\left(\varphi_{0}\right) . \\
& \text { Let } e=(\underbrace{0,0, \ldots, 0,}_{i_{0}-1}, \varphi_{0}, \underbrace{0, \ldots, 0}_{n-i_{0}}) .
\end{aligned}
$$

We prove that

$$
x=A x+\lambda e \text { for } x \in \partial K_{\rho_{0}}^{n} \text { and } \lambda \geq 0 .
$$

In fact, if not, there exist $x \in \partial K_{\rho_{0}}^{n}$ and $\lambda \geq 0$ such that

$$
x=A x+\lambda e .
$$

When $i \neq i_{0}$, we have $x_{i}=A_{i} r x$ and $x_{i} \in K$ by $\left(h_{n}\right)$ with $t=1$. When $i=i_{0}$, by $(E)_{n}, \quad x_{i_{0}}=A_{i_{0}} r x+\lambda \varphi_{0} \geq \lambda \varphi_{0}$. Hence $x \in K^{n}$ and $A+\lambda \hat{e}$ is $r$-nowhere normaloutward map on $\bar{K}_{\rho_{0}}^{n}$ relative to $K^{n}$. This implies $\lambda>0$. By $(E)_{n}$ and (2.4), we have $A_{i_{0}} x \geq \mu_{1}\left(L_{0}\right) L_{0}\left(x_{i_{0}}\right) \geq 0$ and $x_{i_{0}} \geq \lambda \varphi_{0}$. Let

$$
\lambda_{1}=\sup \left\{\lambda>0: x_{i_{0}} \geq \lambda \varphi_{0}\right\} \text {. }
$$


Then $0<\lambda \leq \lambda_{1}<\infty, \quad x_{i_{0}} \geq \lambda_{1} \varphi_{0}$ and $L_{0}\left(x_{i_{0}}\right) \geq \lambda_{1} L_{0}\left(\varphi_{0}\right)=\frac{\lambda_{1}}{\mu_{1}\left(L_{0}\right)} \varphi_{0}$. By $(2.4)$ and $(E)_{n}$,

$$
x_{i_{0}}=A_{i_{0}}(x)+\lambda \varphi_{0} \geq \mu_{1}\left(L_{0}\right) L_{0}\left(x_{i_{0}}\right)+\lambda_{1} \varphi_{0} \geq\left(\lambda_{1}+\lambda\right) \varphi_{0} .
$$

Hence, by (2.5), we have $\lambda_{1} \geq \lambda_{1}+\lambda>\lambda_{1}$, a contradiction. (E) of Theorem 1.1 holds. By Theorem 1.1, $A$ has a fixed point in $\overline{K_{\rho}^{n}} \backslash \bar{K}_{\rho_{0}}$, that is, (2.1) has a solution in $K^{n} \backslash\{0\}$.

The following result shows the nome-type compression and expansion theorem of (2.1).

Theorem 2.2 Let $K$ be a total, normal cone in $X, r: X \rightarrow K$ be a retraction, $A_{i}: K^{n} \rightarrow X$ be compact and satisfy:

$\left(h_{n}\right)^{\prime} \quad t A_{i}$ is r-nowhere normal-outward map with respect to component $x_{i}$ on $K^{n}$ relative to $K$ for any $i \in I_{n}$ and $t \in(0,1]$.

Assume that the following conditions hold: there exist $\rho_{0}, \rho_{1} \in(0, \infty)$ with $\rho_{0}<\rho_{1}$ such that for any $i \in I_{n}$

$(E)_{n} \quad A_{i} x \in K$ for $x \in K_{\rho_{0}}^{n}$ and $\left\|A_{i} x\right\|>\left\|x_{i}\right\|$ for $x \in K_{\rho_{0}}^{n}$ with $\left\|x_{i}\right\|=\rho_{0}$.

$\left(H_{n}\right)\left\|A_{i} x\right\| \leq\left\|x_{i}\right\|$ and $x \in K_{\rho_{1}}^{n}$ with $\left\|x_{i}\right\|=\rho_{1}$.

Then (2.1) has a solution in $K^{n} \backslash\{0\}$.

Proof. Let $A$ be defined by Theorem 2.1 and $x_{i}=0\left(i \in I_{n}\right)$. Then $\left(h_{n}\right)^{\prime}$ implies that $t A$ is $r_{n}$-nowhere normal-outward map on $K^{n}$ relative to $K_{n}$ for $t \in(0,1]$.

Let $x \in \partial K_{\rho_{0}}^{n}$. Then there is $x_{i} \in P$ such that $\left\|x_{i}\right\|=\rho_{0}$ and $\|A x\| \geq\left\|A_{i} x\right\|>$ $\left\|x_{i}\right\|=\rho_{0}=\|x\|$ by $\left(E_{n}\right)$. Since $A: K_{\rho_{0}}^{n} \rightarrow K^{n}$, the standard argument shows $i_{r, K}\left(A, K_{\rho_{0}}^{n}\right)=i_{K}\left(A, K_{\rho_{0}}^{n}\right)=0$, for example, see [6].

Without loss of generality, we may assume that $A$ has no a fixed point in $\partial K_{\rho_{1}}^{n}$ (otherwise, the result has been proved). We prove $x \neq \lambda A x$ for $x \in K_{\rho_{1}}^{n}$ and $0<\lambda \leq 1$.

In fact, if there exist $x \in \partial K_{\rho_{1}}^{n}$ and $0<\lambda \leq 1$ such that $x=\lambda A x$, then $\lambda<1$ since $A$ has no a fixed point in $K_{\rho_{1}}^{n}$. From $x_{i}=\lambda A_{i} x$ for any $i \in I_{n}$, we have $\left\|x_{i}\right\|=\lambda\left\|A_{i} x\right\|$.

Let $i_{0} \in I_{n}$ such that $\left\|x_{i_{0}}\right\|=\rho_{1}$. By $\left(H_{n}\right),\left\|x_{i_{0}}\right\|=\lambda\left\|A_{i_{0}} x\right\|<\left\|A_{i_{0}} x\right\| \leq\left\|x_{i_{0}}\right\|$, it is a contradiction. By Theorem $3.1[3], i_{r, K}\left(A, K_{\rho_{1}}^{n}\right)=1$. 
Hence, $A$ has a fixed point in $K_{\rho_{1}}^{n} \backslash \overline{K_{\rho}^{n}}$ and (2.1) has a solution in $K^{n} \backslash\{0\}$.

Remark 2.1 One may refer to [8] for the nome-type compression and expansion theorem, where maps defined on cone and take values in the cone.

\section{Nonnegative solutions of elliptic boundary value problems}

In this section, we investigate the existence of nonzero nonnegative (classical) solutions of the following elliptic systems:

$$
\left\{\begin{array}{l}
L_{i} u_{i}(z)=f_{i}(z, \mathfrak{u}(z)) \text { in } \Omega \text { and for each } i \in I_{n} \\
u_{i}(z)=0 \text { on } \partial \Omega
\end{array}\right.
$$

where $\Omega \subset \mathrm{R}^{m}(m \in \mathrm{N}, m \geq 2)$ is a bounded domain,

$$
\mathrm{L}_{i} u_{i}(z)=-\sum_{k, j=1}^{m} \frac{\partial}{\partial z_{k}}\left(a_{k j}^{(i)}(z) \frac{\partial u_{i}(z)}{\partial z_{j}}\right)+\sum_{j=1}^{m} b_{j}^{(i)}(z) \frac{\partial u_{i}(z)}{\partial z_{j}}+c^{(i)}(z) u_{i}(z)
$$

where $\mathrm{u}(z)=\left(u_{1}(z), u_{2}(z), \ldots, u_{n}(z)\right), \quad z=\left(z_{1}, z_{2}, \ldots, z_{m}\right), \quad f_{i}: \bar{\Omega} \times \mathrm{R}_{+}^{n} \rightarrow \mathrm{R}$, $f_{i} \in C^{\mu}\left(\Omega \times \mathrm{R}^{n}\right)$ and $\mu \in(0,1)$ is a given constant. When $f_{i} \in C^{\mu}\left(\Omega \times \mathrm{R}^{n}\right)$ $\rightarrow \mathrm{R}_{+}$, system (3.1) was studied in [10]. A single Elliptic BVP with Dirichlet boundary condition was studied in [5]. The definitions of $L$ and $\Omega$ are same as in $[2,10]$.

$\mathrm{u}$ is called to a (classical) solution of (3.1), we mean a function $u_{i} \in C^{2}(\Omega) \cap C(\bar{\Omega})$ satisfying (3.1) pointwise. A solution $\mathrm{u}$ of (3.1) is said to be nonnegative if $u_{i} \in P$, where

$$
P=\{u \in C(\bar{\Omega}): u(z) \geq 0 \quad \text { for } z \in \bar{\Omega}\}
$$

is the positive cone in $C(\bar{\Omega})$, which is total and normal.

We always assume that the following conditions hold for each $i \in I_{n}$ :

$\left(C_{1}\right) a_{k j}^{(i)}, b_{j}^{(i)}, c^{(i)} \in C^{\widehat{\mu}}(\bar{\Omega})$ for $k, j \in I_{m}$, and $c^{(i)}(z) \geq 0$ for $z \in \bar{\Omega}$.

$\left(C_{2}\right) a_{k j}^{(i)}(z)=a_{j k}^{(i)}(z)$ for $z \in \bar{\Omega}$ and $k, j \in I_{m}$, and there exists $\mu_{i}>0$ such that

$$
\sum_{k, j=1}^{m} a_{k j}^{(i)}(z) \xi_{k} \xi_{j} \geq \mu_{i}|\xi|^{2} \text { for } x \in \bar{\Omega} \text { and } \xi=\left(\xi_{1}, \ldots, \xi_{m}\right) \in \mathrm{R}^{m} \text {. }
$$

(C $\left.C_{3}\right) \frac{\partial a_{k j}^{(i)}}{\partial z_{k}} \in C^{\widehat{\mu}}(\bar{\Omega})$ for $k, j \in I_{m}$. 
(C $\left.C_{4}\right) \frac{\partial b_{j}^{(i)}}{\partial z_{j}} \in C(\bar{\Omega})$ and $\frac{\partial b_{j}^{(i)}(z)}{\partial x_{j}} \leq 2 c^{(i)}(z)$ for $x \in \Omega$ and $j \in I_{m}$.

(h) $f_{i} \in C^{\widehat{\mu}}\left(\bar{\Omega} \times \mathbb{R}_{+}^{n}\right)$.

( $\left.h_{2}\right)$ (Positivity condition) $f_{i}\left(z, y_{0}^{i}\right) \geq 0$ for $z \in \Omega$,

where $y_{0}^{i}=\left(y_{1}, y_{2}, \ldots, y_{i-1}, 0, y_{i+1}, \ldots, y_{n}\right)$ and $y_{i} \in \mathrm{R}_{+}$.

Let $L$ be defined by [5] and all assumptions on $L$ hold. Following the known results [5], there is a bounded linear operator $L$ satisfying

(1) $L$ maps $C(\bar{\Omega})$ to $C^{2+\widehat{\mu}}(\bar{\Omega})$.

(2) If $u \in C^{2+\widehat{\mu}}(\bar{\Omega})$ and $v \in C^{\widehat{\mu}}(\bar{\Omega})$ satisfy $u=L v$, then $u$ and $v$ satisfy

see [1, Theorem 4.2].

$$
\left\{\begin{array}{l}
L u(z)=v(z) \text { in } \bar{\Omega} \\
u(z)=0 \text { on } \partial \Omega
\end{array}\right.
$$

Let $\psi \in C^{\widehat{\mu}}(\bar{\Omega})$ and let

$$
L_{\psi}(u)=L(\psi u) \text { for } u \in C(\bar{\Omega})
$$

and

$$
\underline{\psi}=\min \{\psi(z): z \in \bar{\Omega}\} .
$$

It is well known that if $\psi \in C^{\widehat{\mu}}(\bar{\Omega})$ with $\psi>0$, then $L_{\psi}: C(\bar{\Omega}) \rightarrow C^{\sigma}(\bar{\Omega}) \subset$ $C(\bar{\Omega})$ is a compact linear operator such that $\bar{L}_{\psi}(P) \subset P$ for each $\sigma \in(0,2)$ and there exists $\varphi \in P \cap{C_{0}}^{2+\widehat{\mu}}(\bar{\Omega}) \backslash\{0\}$ such that

$$
\varphi=\mu_{1}\left(L_{\psi}\right) L_{\psi} \varphi,
$$

where $\mu_{1}\left(L_{\psi}\right)=1 / r\left(L_{\psi}\right)$ and $r\left(L_{\psi}\right)$ is the spectral radius of $L_{\psi}$, see [1, Theorem 4.2].

We define an operator $A_{i}: P^{n} \rightarrow C(\bar{\Omega})$ by

$$
\left(A_{i} \mathrm{u}\right)(z)=\left(L_{i} F_{i} \mathrm{u}\right)(z)
$$

where $L_{i}$ is an operator corresponding to $L$ when $L=L_{i}$ in (3.4) and $F_{i}$ : $P^{n} \rightarrow C(\bar{\Omega})$ is a Nemytskii operator defined by 


$$
\left(F_{i} \mathrm{u}\right)(z)=f_{i}(z, \mathrm{u}(z))
$$

Let $A: P^{n} \rightarrow C(\bar{\Omega})_{n}$ by $A \mathrm{u}=\left(A_{1} \mathrm{u}, A_{2} \mathrm{u}, \ldots, A_{n} \mathrm{u}\right)$.

By the known results, it is easy to verify under the conditions $\left(h_{1}\right)$ that $A$ is compact, and $\mathrm{u} \in P^{n}$ is a solution of the following fixed point equation

$$
\mathrm{u}(z)=A \mathrm{u}(z) \text { for } z \in \bar{\Omega},
$$

if and only if $u_{i} \in C_{0}{ }^{2+\widehat{\mu}}(\bar{\Omega})$ and $\mathrm{u}$ is a nonnegative solution of (3.1), see [5, Lemma 2.2].

Let $r: C(\bar{\Omega}) \rightarrow P$ be defined by

$$
r(u)(z)=u^{+}(z):=\max \{u(z), 0\} .
$$

Then it is easy to know that $r$ is a retraction from $C(\bar{\Omega})$ to $P, r^{-1}(P)=C(\bar{\Omega})$ and $r: C(\bar{\Omega}) \rightarrow P$ is a Lipschitz continuous map with Lipschitz constant 1 .

Theorem 3.1 Assume that $\left(C_{4}\right),\left(h_{1}\right)$ and $\left(h_{2}\right)$ hold. Then $t A$ is a $r_{n}$-nowhere normal-outward map on $P^{n}$ relative to $P^{n}$ for $t \in(0,1]$, where $A_{i}$ and $r$ are same as in (3.7) and (3.10), respectively.

Proof. Let $\mathrm{u}=\left(u_{1}, u_{2}, \ldots, u_{i}, \ldots, u_{n}\right) \in C(\bar{\Omega})_{n}$ such that $\mathrm{u}(z)=t A\left(r_{n} \mathrm{u}\right)(z)$. Then $\mathrm{u}_{i}(z)=t A_{i}\left(r \mathrm{u}_{i}\right)(z)$ for any $i \in I_{n}$. Similarity to the proof of Theorem 3.1 [5], we obtain $u_{i} \in P$ and $\mathrm{u} \in P^{n}$.

By using Theorems 2.1 and 3.1, we prove our main result on the existence of nonzero nonnegative solutions of (3.1).

Theorem 3.2 Assume that $\left(C_{4}\right),\left(h_{1}\right),\left(h_{2}\right)$ and the following conditions hold.

$\left(H_{1}\right)$ For any $i \in I_{n}$, there exist $\phi_{i} \in C^{\widehat{\mu}}(\bar{\Omega})$ with $\underline{\phi_{i}}>0, \quad \varepsilon_{i} \in\left(0, \mu_{1}\left(L_{\phi_{i}}\right)\right)$ and $u_{0}^{i} \in P$ such that

$$
f_{i}(z, y) \leq u_{0}^{i}(z)+\left(\mu_{1}\left(L_{\phi_{i}}\right)-\varepsilon_{i}\right) \phi_{i}(y) y_{i} \text { for } z \in \bar{\Omega} \text { and } y \in \mathrm{R}_{+}^{n} .
$$

( $\left.H_{2}\right)$ There exists $i_{0} \in I_{n}$ and $\psi_{\rho} \in C^{\widehat{\mu}}(\bar{\Omega})$ with ${\underline{\psi_{\rho}}}>0$ such that

$$
f_{i_{0}}(z, y) \geq \mu_{1}\left(L_{\psi_{\rho}}\right) \psi_{\rho}(y) y_{i_{0}} \text { for } z \in \bar{\Omega},|y| \in[0, \rho] \text { and } y \in \mathbf{R}_{+}^{n} \text {. }
$$


Then (3.1) has a solution $\mathrm{u} \in P^{n} \backslash\{0\}$ with $u_{i} \in C_{0}{ }^{2+\widehat{\mu}}(\bar{\Omega})$.

Proof. By Theorem 3.1, we have that $t A$ is a $r_{n}$-nowhere normal-outward map on $P^{n}$ relative to $P^{n}$ for $t \in(0,1]$.

Since $L_{i}(P) \subset P$, by $\left(H_{1}\right)$ we have

$$
A_{i} \mathrm{u}(z) \leq u_{1}^{i}(z)+\left(\mu_{1}\left(L_{\phi_{i}}\right)-\varepsilon\right) L_{\phi_{i}}\left(u_{i}\right) \text { for } \mathrm{u} \in P^{n} \text { and } z \in \bar{\Omega} \text {. }
$$

where $u_{1}^{i}(z)=L\left(u_{0}^{i}\right)(z) \geq 0$ for $z \in \bar{\Omega}$. Hence, $A$ satisfies Theorem $2.1(L S)_{n}$. By $\left(H_{2}\right)$ and $L_{i_{0}}(P) \subset P$, we have

$$
A_{i_{0}} \mathrm{u}(z) \geq \mu_{1}\left(L_{\psi_{\rho}}\right) L_{\psi_{\rho}}\left(u_{i_{0}}\right) \text { for } \mathrm{u} \in \partial P_{\rho}^{n} \text { and } z \in \bar{\Omega} .
$$

and $A$ satisfies Theorem $2.1(E)_{n}$.

By Theorem 2.1, there exists $\mathrm{u} \in P^{n} \backslash P_{\rho}^{n}$ such that $\mathrm{u}=A \mathrm{u}$. It follows that $\mathrm{u}$ is a solution of (3.1) and $u_{i} \in C_{0}{ }^{2+\widehat{\mu}}(\bar{\Omega})$.

Notation: Let $\psi_{i}: \bar{\Omega} \rightarrow(0, \infty)$ with $\underline{\psi_{i}}>0$. For $y_{i} \in\left(R^{+} \backslash\{0\}\right)$, let

$$
\begin{gathered}
\underline{f_{\psi_{i}}}\left(y_{i}\right)=\inf _{(z, y) \in \bar{\Omega} \times R_{+}^{n}} \frac{f_{i}(z, y)}{\psi_{i}(z)}, \overline{f_{\psi_{i}}}\left(y_{i}\right)=\sup _{(z, y) \in \bar{\Omega} \times R_{+}^{n}} \frac{f_{i}(z, y)}{\psi_{i}(z)}, \\
\left(f_{\psi_{i}}\right)_{0}=\lim _{y_{i} \rightarrow 0+} \inf \underline{f_{\psi_{i}}}\left(y_{i}\right) / y_{i},\left(f_{\psi_{i}}\right)^{\infty}=\lim _{y_{i} \rightarrow \infty} \sup \overline{f_{\psi_{i}}}\left(y_{i}\right) / y_{i} .
\end{gathered}
$$

As a special case of Theorem 3.2, we obtain the following result.

Corollary 3.1 Assume that $\left(C_{4}\right),\left(h_{1}\right),\left(h_{2}\right)$ and there exists $\psi_{i} \in C^{\widehat{\mu}}(\bar{\Omega})$ with $\underline{\psi_{i}}>0$ such that the following condition holds.

$\left(H_{1}\right)^{\prime}\left(f_{\psi_{i}}\right)^{\infty}<\mu_{1}\left(L_{\psi_{i}}\right)$ for any $i \in I_{n}$.

$\left(H_{2}\right)^{\prime} \quad \mu_{1}\left(L_{\psi_{i_{0}}}\right)<\left(f_{\psi_{i_{0}}}\right)_{0}$ for some $1 \leq i_{0} \leq n$.

$\left(H_{3}\right)$ For any $r \in(0, \infty)$ and $i \in I_{n}$, there is $M_{r}^{i}>0$ such that $f_{i}(z, y) \leq M_{r}^{i}$ for $z \in R_{+}^{n}$ and $y_{i} \in[0, r]$.

Then (3.1) has a solution $\mathrm{u} \in P^{n} \backslash\{0\}$ with $u_{i} \in C_{0}{ }^{2+\widehat{\mu}}(\bar{\Omega})$.

Proof. By $\left(H_{1}\right)^{\prime}$ and $\left(H_{3}\right)$, there exists $\varepsilon_{i}>0$ such that

$$
f_{i}(z, y) \leq\left(\mu_{1}\left(L_{\psi_{i}}\right)-\varepsilon_{i}\right) \psi_{i}(z) y_{i} \quad \text { for }(z, y) \in \bar{\Omega} \times R_{+}^{n} \text { and } y_{i} \in\left[r_{i}, \infty\right)
$$

and

$$
f_{i}(z, y) \leq M_{r_{i}}^{i}+\left(\mu_{1}\left(L_{\psi_{i}}\right)-\varepsilon_{i}\right) \psi_{i}(z) y_{i} \quad \text { for }(z, y) \in \bar{\Omega} \times R_{+}^{n} .
$$

By $\left(H_{2}\right)^{\prime}$, it is easy to see that there exists $\rho>0$ such that $f_{i}(z, y) \geq 0$ for $z \in \bar{\Omega},\|y\| \leq \rho$ for every $i \in I_{n}$ and 


$$
f_{i_{0}}(z, y) \geq \mu_{1}\left(L_{\psi_{i_{0}}}\right) \psi_{i_{0}}(z) y_{i_{0}} \quad \text { for }(z, y) \in \bar{\Omega} \times R_{+}^{n} \text { and }|y| \in[0, \rho]
$$

The result follows from Theorem 3.2.

Remark 3.1 Let $f_{i}(z, y)=\psi_{i}(z) y_{i} e^{\alpha_{i}-\sum_{j=1}^{n} \beta_{i j} y_{j}-\frac{m_{i}}{1+\sum_{j=1}^{n} k_{i j} y_{j}}}-d_{i} y_{i}^{\sigma_{i}}$ or $\quad f_{i}(z, y)=$ $\frac{s_{i} y_{i}^{\gamma_{i i}}}{1+y_{i}^{\gamma_{i i}}+\sum_{j=1, j \neq i}^{n} a_{j} y_{j}^{\gamma_{i j}}}-d_{i} y_{i}^{\sigma_{i}}$, all the parameters are nonnegative, $\sigma_{i} \geq 1$.

When $\sigma_{i}=1$, population models of Ricker types (3.1) and Beverton-Holt types (4.1) in [10] were studied. However, the results can be not utilized to discuss a case of $\sigma_{i}>1$, but we can treat the case of $\sigma_{i}>1$ since $f_{i}\left(z, y_{0}^{i}\right) \geq 0$.

\section{Conclusions and Remarks}

We study the existence of nonnegative solutions of a nonlinear system consisting of nowhere normal-outward maps in Banach spaces, this type of maps defined on a cone does not necessarily take values in the cone and contains weakly inward maps and generalized inward maps under the appropriate conditions [3]. In history, someone established the fixed point index of weakly inward maps or generalized inward maps, but it is difficult to use the index since verifying the weakly inward maps or generalized inward maps is very difficult [9]. From the discussion in this paper, we know that verifying the nowhere normal-outward map is easy in applications. The existence of nonnegative solutions [5] of a single elliptic BVP is extended to system (3.1) via using Theorem 2.1, system (3.1) has many uses such as population models [10] or Volterra-Lotka competition models [11] or steady state periodic solutions for parabolic systems [12]. We hope that the results of this paper will be applied in many fields.

ACKNOWLEDGEMENTS This work was supported in part the Applied Basic Research Project of Sichuan Province (No. 2018JY0169) 


\section{References}

[1] Amann, H. (1976). Fixed point equations and nonlinear eigenvalue problems in ordered Banach spaces, SIAM. Rev. 18, 620-709.

[2] Lan, K. Q. (2011). Nonzero positive solutions of systems of elliptic boundary value problems, Proc. Amer. Math. Soc. 139, 4343-4349.

[3] Yang, G. C. and Lan, K. Q. (2016). A fixed point index theory for nowhere normaloutward compact maps and applications, J. Appl. Anal. Comput. 6, 665683.

[4] Halpern, B. and Bergman, G. (1968). A fixed point theorem for inward and out wardmaps, Trans.Amer.Math.Soc. 130, 353-358.

[5] Lan, K. Q. and Lin, W. (2020). Positive solutions of elliptic boundary value problems and applications to population dynamics, J. Dyn. Differ. Equ. 32, 873-894.

[6] Deimling, K. (1985). Nonlinear Functional Analysis, Spinger-Verlag, New York.

[7] Krein, M. G. and Rutman, M. A. (1962). Linear operators leaving invariant a cone in a Banach space, Amer. Math. Soc. Transl. 10, 199-325.

[8] Guo, D. and Lakshmikantham, V. (1988). Nonlinear Problems in Abstract Cones, Academic Press, New York.

[9] Webb, J. R. L. and Lan, K. Q. (2006). Eigenvalue criteria for existence of multiple positive solutions of nonlinear boundary value problems of local and nonlocal type, Topol. Methods Nonlinear Anal. 27, 91-116.

[10] Lan, K. Q. and Lin, W. (2019). Systems of elliptic boundary value problems and applications to competition models, Applied. Math. Lett. 90, 86-92.

[11] Cosner, C. and Lazer, A. C. (1984). Stable coexistence states in the VolterraLotka competition models with diffusion, SIAM. J. Appl. Math. 44, 1112-1132.

[12] Wang, C. Y. (2008). Existence and stability of periodic solutions for parabolic systems with time delays, J. Math. Anal. Appl. 339, 1354-1361. 